\title{
Graded histologic response after neoadjuvant chemotherapy is an optimal criterion for treatment change in patients with locally advanced gastric cancer
}

\author{
Xiang Wang ${ }^{1 \#}$, Xiaoyi Li $^{2 \#}$, Na Zhou ${ }^{1}$, Dingrong Zhong ${ }^{3}$, Chunmei Bai ${ }^{1}$, Lin Zhao ${ }^{1}$ \\ ${ }^{1}$ Department of Medical Oncology, ${ }^{2}$ Department of General Surgery, Peking Union Medical College Hospital, Chinese Academy of Medical \\ Sciences, Beijing 100730, China; ${ }^{3}$ Department of Pathology, China-Japan friendship Hospital, Beijing 100029, China \\ Contributions: (I) Conception and design: X Wang, L Zhao; (II) Administrative support: C Bai; (III) Provision of study materials or patients: X \\ Li, N Zhou; (IV) Collection and assembly of data: X Wang, X Li, L Zhao; (V) Data analysis and interpretation: X Wang, D Zhong, L Zhao; (VI) \\ Manuscript writing: All authors; (VII) Final approval of manuscript: All authors. \\ \#These authors contributed equally to this work. \\ Correspondence to: Lin Zhao. Department of Medical Oncology, Peking Union Medical College Hospital, Chinese Academy of Medical Sciences, \\ No. 1 Shuai Fu Yuan, Dongcheng District, Beijing 100730, China. Email: wz20010727@aliyun.com.
}

\begin{abstract}
Background: The necessity for changing the postoperative therapy regimen for locally advanced gastric cancer after ineffective neoadjuvant chemotherapy and surgery is unclear because there are no criteria to determine which patients can benefit from this treatment. We assessed whether graded histologic regression of $<50 \%$ could be the criterion for regimen modification.

Methods: The study was designed as a matched-pair case-control investigation to minimize intergroup heterogeneity. Patients were stratified into two groups in which they either continued in the same course of treatment or changed the regimen to neoadjuvant chemotherapy.

Results: Thirty-six patients were stratified into two groups. The adjuvant chemotherapy regimen of 12 patients was changed, while 24 patients continued on the same regimen. During an average follow-up period of 36 months, there was no difference observed in overall survival in the two groups (median, $24.0 \mathrm{vs}$. 31.0 months, $\mathrm{P}=0.863$ ). In a subgroup analysis, however, patients in the changed regimen group with ypTNM stage III disease tended to have superior overall survival, though this effect was not significant (median, 23.0 vs. 14.0 months, $\mathrm{P}=0.123$ ). Post-therapy nodal status was associated with overall survival in the multivariate analysis ( $\mathrm{P}=0.014$, HR 12.503, 95\% CI: 1.664-93.919). Most adverse events were categorized as grade 1 or 2 , and all treatments were well tolerated.

Conclusions: Changing treatment based on a graded histologic regression of $<50 \%$ after neoadjuvant chemotherapy did not prolong overall survival in patients with gastric cancer. However, changing the adjuvant regimen did reveal a trend towards improved overall survival in the ypTNM stage III subgroup, which merits further investigation using a larger sample size.
\end{abstract}

Keywords: Treatment change; graded histologic response; neoadjuvant chemotherapy; advanced gastric cancer

Submitted Sep 03, 2019. Accepted for publication Sep 09, 2019.

doi: $10.21037 /$ atm.2019.09.82

View this article at: http://dx.doi.org/10.21037/atm.2019.09.82 


\section{Introduction}

Gastric cancer is one of the most common malignant cancers worldwide, and is the second most common cancer in China, with incidences increasing each year and a mortality rate second only to that of lung cancer (1). Surgery is the primary treatment for gastric cancer. Due to a lack of screening, gastric cancer is rarely detected early in China. Approximately $50 \%$ of patients with gastric cancer are diagnosed at an advanced stage, leading to a low radical resection rate and a high postoperative relapse rate. New treatments are necessary to improve the resection rate, especially the radical resection rate, to improve the prognosis of gastric cancer.

In recent years, the advent of neoadjuvant chemotherapy has helped to downstage the primary tumour, facilitate complete surgical resection, eliminate micrometastases, and test the sensitivity of the tumor to chemotherapy. Several clinical investigations (2-4) and a meta-analyses (5) have shown that preoperative chemotherapy for gastric cancer, or neoadjuvant chemotherapy, could improve the R0 resection rate and the survival of patients with advanced gastric cancer. Nevertheless, further clinical studies are needed to solve problems in this field, including proper population selection, accurate preoperative staging, the selection of a preoperative chemotherapy regimen, efficacy criteria for preoperative chemotherapy, the need for postoperative adjuvant chemotherapy in case of effective neoadjuvant chemotherapy, and the selection of a postoperative adjuvant chemotherapy regimen in case of ineffective neoadjuvant chemotherapy.

The histologic response to neoadjuvant chemotherapy helps to assess the sensitivity of the tumor to chemotherapy. Accurate histological evaluation aids in determining the effect of preoperative chemotherapy on the tumor and consequently, provides essential information for the selection of a postoperative chemotherapy regimen. Currently, many studies have shown that the histological evaluation of neoadjuvant chemotherapy is related to the survival of patients with gastric cancer (6-11). Moreover, a univariate analysis in our study (11) showed that patients with a graded histologic regression (GHR) of the primary tumor $\geq 50 \%$ survived longer than patients with a GHR of $<50 \%$. This finding suggests that a GHR of $<50 \%$ may indicate a poor response to preoperative chemotherapy and need a different postoperative treatment regimen.

We conducted a retrospective matched-pair case-control analysis of patients with locally advanced gastric cancer who underwent neoadjuvant chemotherapy in the Peking
Union Medical College Hospital between December 2006 and September 2012. Specifically, this study investigated the needs and criteria for modifying the post-gastrectomy chemotherapy regimen based on the patient's response to preoperative chemotherapy to further improve patient survival.

\section{Methods}

\section{Patient selection}

We conducted a retrospective matched-pair case-control analysis to minimize inter-group differences. All patients were confirmed to have gastric or gastroesophageal junction adenocarcinoma based on an endoscopic biopsy and met the following criteria: (I) TNM stage of T2$\mathrm{T} 4$ or positive regional lymph nodes, according to the American Joint Committee on Cancer (AJCC) 7.0 staging system, verified by enhanced abdominal computed tomography (CT) and/or endoscopic ultrasound (EUS), positron emission tomography (PET)/CT scan, with no evidence of distant metastases; (II) ECOG performance status score $\leq 2$ without serious heart, lung, liver, kidney, or hematological dysfunctions; (III) age $\geq 18$ years old; (IV) no previous chemotherapy, radiotherapy, or surgical treatment for gastric cancer; (V) no contraindications for receiving oxaliplatin-based neoadjuvant chemotherapy; (VI) gastrectomy was performed after preoperative chemotherapy if imaging studies did not confirm disease progression [according to the Response Evaluation Criteria in Solid Tumors (RECIST)] (12); and (VII) signed the informed consent form.

From December 2006 to September 2012, a total of 89 patients who received oxaliplatin-based neoadjuvant chemotherapy were enrolled in the study, and 74 of these patients underwent a radical gastrectomy. All patients with a postoperative GHR of the primary tumor $<50 \%$ were matched for gender, age, primary tumor site, tumor differentiation, and TNM staging before neoadjuvant chemotherapy Patients were then paired at a 2:1 ratio. A total of 36 patients met the inclusion criteria, and 24 of these patients continued to receive the original neoadjuvant chemotherapy, while 12 patients received a modified chemotherapy regimen after surgery.

\section{Treatment schedule}

All 36 enrolled patients were treated with oxaliplatinbased neoadjuvant chemotherapy. Among them, 34 
(94.4\%) received a modified 5-fluorouracil (5-FU) (Yabao Pharmaceutical, Shanxi, China)/leucovorin (HengRui Medicine Co., Ltd., Jiangsu, China)/oxaliplatin (SanofiAventis, Paris, France) (mFOLFOX6) regimen, and 2 (5.6\%) received capecitabine (Roche, Basel, Switzerland)/oxaliplatin (XELOX) neoadjuvant chemotherapy. All patients underwent radical resection after 3 cycles of chemotherapy. Postoperative chemotherapy was started 3-4 weeks after surgery, and the regimen was chosen at their physicians' discretion. Twelve patients received modified taxane-based chemotherapy, 8 of whom $(66.7 \%)$ received the docetaxel (Sanofi-Aventis, Paris, France)/cisplatin (Hospira Australia Pte Ltd., Lake Forest, America)/5-FU (DCF) regimen, 2 (16.7\%) received the paclitaxel (Bristol-Myers Squibb, New York, NY, USA)/carboplatin (Bristol-Myers Squibb, New York, NY, USA) regimen, 1 (8.3\%) received the paclitaxel/ oxaliplatin/capecitabine regimen, and $1(8.3 \%)$ received the paclitaxel + capecitabine regimen. The entire duration of pre- and postoperative chemotherapies was 6 months, and the cycles of postoperative chemotherapy were planned due to different protocols.

\section{Histological evaluation criteria}

The histological evaluation after preoperative therapy was assessed by an independent pathologist (DRZ) who evaluated all specimens from radical surgery and confirmed the proportion of tumor necrosis and fibrosis within the lesion. The percentage of residual tumor cells, or GHR, within the lesion was recorded as $0-100 \%$, with $0 \%$ representing no necrosis or cellular or structural changes within the whole lesion and 100\% having an entire lesion that disappeared or replaced by fibrous tissue without any viable tumor cells.

\section{Follow-up}

Up until January $31^{\text {st }}, 2016$, patients were followed up with at regular intervals (every 3 to 6 months) by either a clinic visit or by telephone. Tumor markers and CT scans were performed regularly (13).

\section{Statistical analysis}

SPSS17.0 software (SPSS Inc., Chicago, IL, USA) was used for all statistical analyses. Overall survival (OS) was defined as the time from the first dosing of preoperative chemotherapy to the time of all-cause death. Progression- free survival (PFS) was defined as the time from the first dosing of preoperative chemotherapy to disease progression confirmed with imaging studies or the pathological examination of the surgical specimen. The chi-squared test was performed to compare counted data between the two groups. The t-test was performed to compare measured data (with normal or near-normal distributions) between the two groups. Kaplan-Meier survival curves were used to analyze survival and progression, and a log-rank test was performed to compare the survival rate between the two groups. A Cox proportional hazards regression model was used for the multivariate analysis. Moreover, the Cox model was used to analyze the effects of different treatments by subgroup. All tests and $\mathrm{P}$ values were two-sided, and $\mathrm{P}<0.05$ was considered statistically significant.

\section{Results}

\section{Patients}

All 36 patients with a postoperative GHR of $<50 \%$ in the primary tumour were treated with oxaliplatin-based neoadjuvant chemotherapy, then underwent gastrectomy with D2 lymphadenectomy (R0 resection), and after, began adjuvant chemotherapy 3 to 4 weeks after surgery. Patients in group A $(n=24)$ continued to receive the original neoadjuvant chemotherapy, while patients in group B $(n=12)$ received modified chemotherapy. Group A included 18 males and 6 females (male: female $=3: 1$ ) aged $58 \pm 9.7$ years, with an ECOG score of 0 to 1 . Group B included 10 males and 2 females (male: female $=5: 1$ ) aged $51 \pm 15$ years, with an ECOG score of 0 to 1 . The basic information and matching of the two groups are shown in Table 1. Postoperative pathological data are shown in Table 2.

In group A, 15 patients (62.5\%) completed postoperative adjuvant chemotherapy as planned; 5 patients $(20.8 \%)$ declined further chemotherapy after 6, 2, 2, 1, or 1 cycles of chemotherapy due to their Grade III nausea and vomiting; 1 patient (4.2\%) discontinued chemotherapy after 3 cycles of chemotherapy because of disease progression; 3 patients $(12.5 \%)$ declined further chemotherapy after 6 , 4 , or 1 cycles of chemotherapy for other reasons. In group B, 9 patients (75.0\%) completed postoperative adjuvant chemotherapy as planned; 2 patients $(16.7 \%)$ declined further chemotherapy after 1 or 2 cycles of chemotherapy because of Grade III nausea and vomiting; and 1 patient $(8.3 \%)$ discontinued chemotherapy after 4 cycles of chemotherapy because of disease progression. 
Table 1 Baseline demographics and clinical characteristics

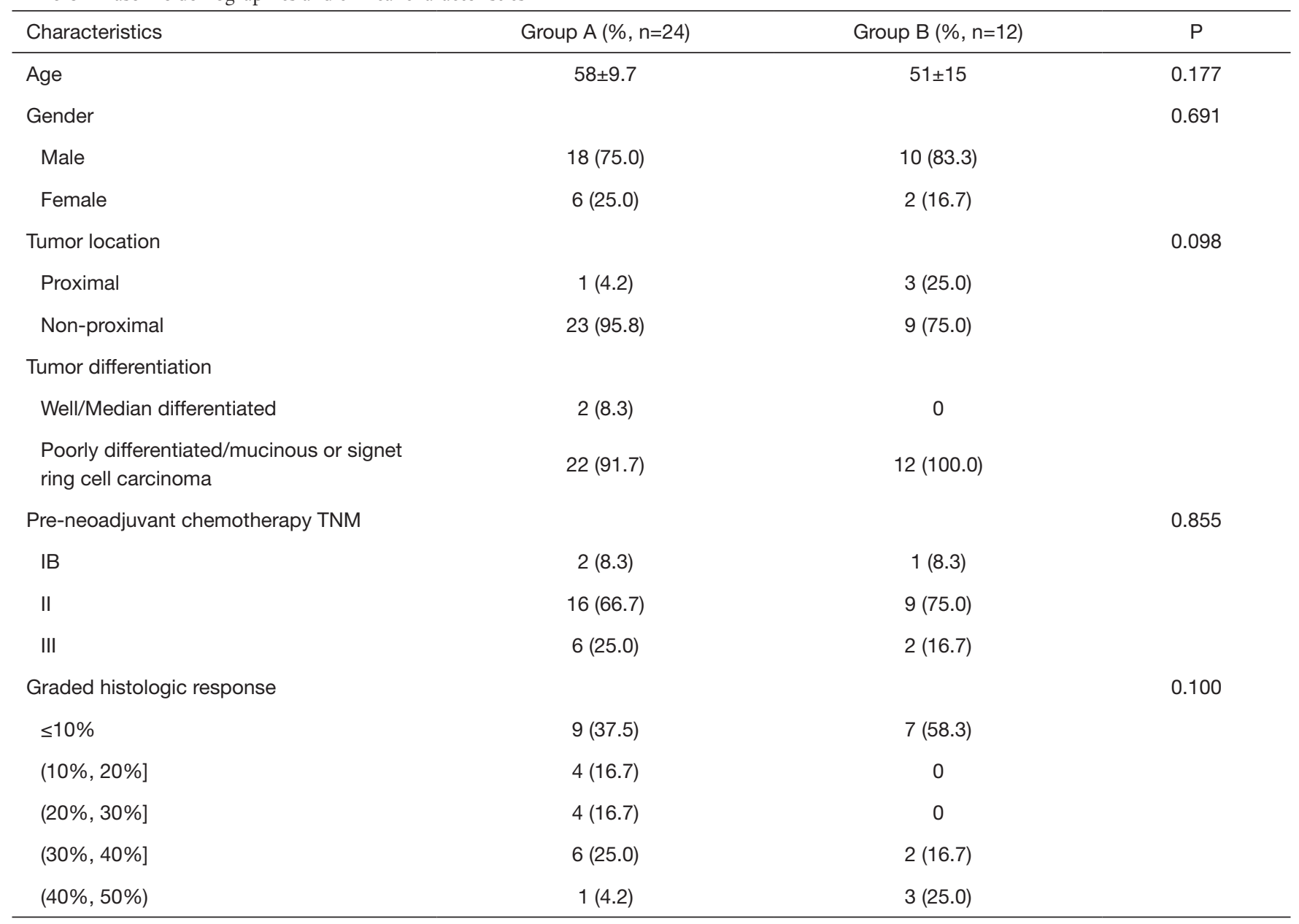

\section{Adverse events}

In both groups, adverse reactions were categorized as grade 1 or 2 . However, the incidence of hematological and non-hematological adverse reactions was significantly higher in group B than in group A. In both groups, the most common grade 3-4 hematological toxicity was neutropenia, with incidence rates of $20.8 \%$ in group A and $41.7 \%$ in group B. Moreover, 1 patient in group B suffered from febrile neutropenia. In both groups, the most common grade 3-4 non-hematological toxicity was nausea and vomiting, with the incidence of $4.2 \%$ in group A and $8.3 \%$ in group B. No chemotherapy-related deaths were observed in this study (Table 3).

\section{Survival}

The follow-up time ranged from 6.0 to 104.0 months, with a median of 36.0 months. Thirteen patients $(54.2 \%)$ in group A experienced disease progression or died. Among group A patients with disease progression, 1 patient (4.2\%) received palliative radiotherapy, 1 patient $(4.2 \%)$ received palliative chemotherapy, and the remaining patients received optimal supportive care alone. In group B, 7 patients $(58.3 \%)$ experienced disease progression or died. Among the group B patients with disease progression, 3 patients $(25.0 \%)$ received palliative chemotherapy, and the remaining patients received optimal supportive care alone.

The median progression-free survival (mPFS) was longer in group A than in group B, but this difference was not significant (26.0 vs. 19.0 months, $\mathrm{P}=0.921$ ) (Figure 1A). The median overall survival (mOS) was also longer in group A than in group $\mathrm{B}$, but this difference was not significant $(31.0$ vs. 24.0 months, $\mathrm{P}=0.863$ ) (Figure $1 B$ ).

Univariate analysis was performed for the clinicopathologic 
factors that might influence survival (Table 4). The results proved that only postoperative $\mathrm{N}$ staging was correlated with OS (Figure 1C). Multivariate analysis was performed by incorporating the factors of primary tumor site, tumor differentiation, TNM staging before neoadjuvant chemotherapy, postoperative $\mathrm{T}$ staging, postoperative $\mathrm{N}$ staging, and postoperative chemotherapy (original vs. modified regimen) with COX regression. The results demonstrated that only postoperative $\mathrm{N}$ staging had a statistically significant association with OS

Table 2 Pathologic features of the resected specimens

\begin{tabular}{lcc}
\hline Characteristics & Group A $(\%, \mathrm{n}=24)$ & Group B (\%, $\mathrm{n}=12)$ \\
\hline ypT stage & $7(29.2)$ & $2(16.7)$ \\
T1-2 & $17(70.8)$ & $10(83.3)$ \\
T3-4 & & \\
ypN stage & $10(41.7)$ & $1(8.3)$ \\
N- & $14(58.3)$ & $11(91.7)$ \\
N+ & & \\
ypTNM & $4(16.7)$ & 0 \\
I & $9(37.5)$ & $4(33.3)$ \\
II & $11(45.8)$ & $8(66.7)$ \\
III & & \\
\hline
\end{tabular}

( $\mathrm{P}=0.014$, HR 12.503, 95\% CI: 1.664-93.919).

\section{Subgroup analysis}

The 36 patients were grouped according to the postoperative TNM staging. While receiving the original neoadjuvant regimen or modified regimen, patients with stage I or stage II had not yet reached the mPFS $(\mathrm{P}=0.904)$ or $\mathrm{mOS}(\mathrm{P}=0.998)$ after postoperative chemotherapy. Neither the mPFS nor the mOS significantly differed between the two groups. Among patients with stage III disease, the mPFS was 11.0 months in patients who received the original neoadjuvant chemotherapy after surgery, which was shorter than the mPFS of patients who received modified chemotherapy after surgery (16.3 months). There was a significant prolongation in the modified chemotherapy arm, though the difference was not statistically significant $(\mathrm{P}=0.207)$ (Figure 1D). The mOS of patients who received the original neoadjuvant chemotherapy after surgery was 14.0 months, which was shorter than that of patients who received modified chemotherapy after surgery (23.0 months). Although there was no significant difference, the curve was separated $(\mathrm{P}=0.123)$ (Figure $1 E)$.

Furthermore, the patients were divided into several groups: GHR $\leq 10 \%$ vs. $>10 \%$, GHR $\leq 20 \%$ vs. $>20 \%$, GHR $\leq 30 \%$ vs. $>30 \%$, and GHR $\leq 40 \%$ vs. $>40 \%$. Neither the PFS nor the OS significantly differed between patients who received the

Table 3 Adverse events with adjuvant chemotherapy

\begin{tabular}{|c|c|c|c|c|}
\hline \multirow{2}{*}{ Adverse event } & \multicolumn{2}{|c|}{ Group A $(n=24)(\%)$} & \multicolumn{2}{|c|}{ Group B (n=12) (\%) } \\
\hline & All grade & Grade 3/4 & All grade & Grade $3 / 4$ \\
\hline Leucocytes & $12(50.0)$ & $4(16.7)$ & $10(83.3)$ & $5(41.7)$ \\
\hline Neutropenia & $8(33.3)$ & $5(20.8)$ & $10(83.3)$ & $5(41.7)$ \\
\hline Anemia & $3(12.5)$ & 0 & $4(33.3)$ & 0 \\
\hline Febrile neutropenia & 0 & 0 & $1(8.3)$ & 0 \\
\hline Thrombocytopenia & $3(12.5)$ & $1(4.2)$ & $3(25.0)$ & 0 \\
\hline Nausea & $12(50.0)$ & $1(4.2)$ & $8(66.7)$ & $1(8.3)$ \\
\hline Vomiting & $4(16.7)$ & $1(4.2)$ & $6(50.0)$ & $1(8.3)$ \\
\hline Anorexia & $15(62.5)$ & 0 & $8(66.7)$ & 0 \\
\hline Diarrhea & $2(8.3)$ & 0 & $2(16.7)$ & 0 \\
\hline Fatigue & $5(20.8)$ & 0 & $5(41.7)$ & 0 \\
\hline Weight loss & $2(8.3)$ & 0 & $3(25.0)$ & 0 \\
\hline Peripheral neuropathy & $5(20.8)$ & 0 & $2(16.7)$ & 0 \\
\hline Drug Fever & $1(4.2)$ & 0 & 0 & 0 \\
\hline
\end{tabular}



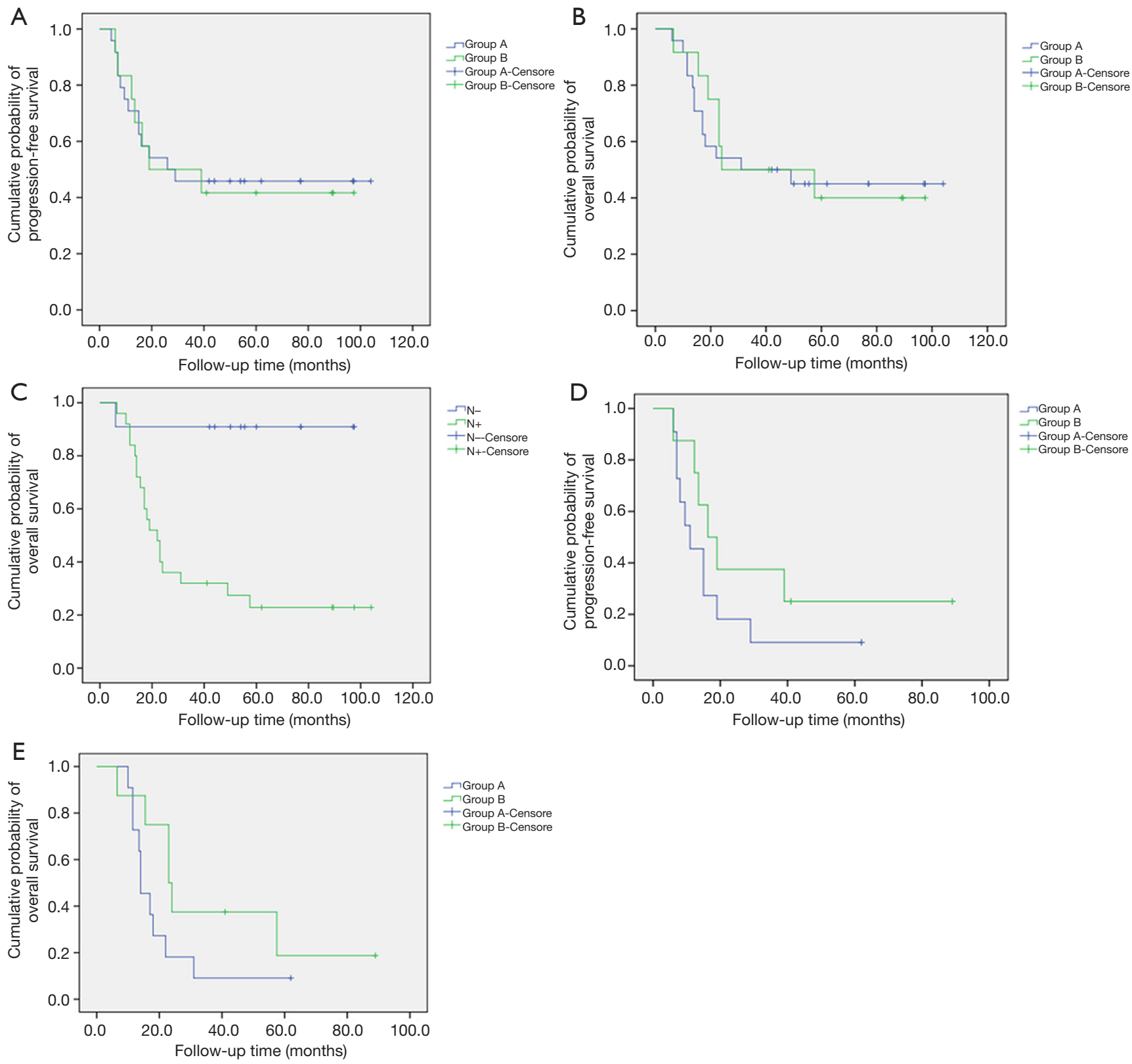

Figure 1 Prognostic value of the graded histologic response. Kaplan-Meier plots for PFS (A) and OS (B) of patients who continued to receive the original neoadjuvant chemotherapy based on GHR <50\% (Group A, n=24) compared with those of patients who received modified chemotherapy (Group B, n=12). OS (C) by post-therapy nodal status (n=36). PFS (D) and OS (E) of patients who continued to receive the original neoadjuvant chemotherapy based on ypTNM stage III (Group A, n=11) compared with those of patients who received modified chemotherapy (Group B, n=8). OS, overall survival.

original chemotherapy and those who received modified chemotherapy. Nevertheless, in the GHR $\leq 10 \%$ vs. $>10 \%$ and GHR $\leq 20 \%$ vs. $>20 \%$ groups, the PFS and the OS of patients who received modified chemotherapy were longer than those of patients who continued to receive the original chemotherapy. In both the GHR $\leq 10 \%$ and GHR $\leq 20 \%$ groups, the PFS was 15.0 months, and the OS was 18.0 months in patients who continued to receive the original chemotherapy. Meanwhile, the PFS and OS were 19.0 and 24.0 months, respectively, in patients who received modified 
Table 4 Univariate analysis of prognostic factors for OS

\begin{tabular}{|c|c|c|c|}
\hline Prognostic factor & $\mathrm{n}=36$ & MST (months) & $\mathrm{P}$ \\
\hline Gender & & & 0.605 \\
\hline Male & 28 & 49.0 & \\
\hline Female & 8 & 18.0 & \\
\hline Age & & & 0.970 \\
\hline$\geq 60$ & 18 & 24.0 & \\
\hline$<60$ & 18 & 31.0 & \\
\hline Location & & & 0.612 \\
\hline Proximal & 4 & 57.5 & \\
\hline Non-proximal & 32 & 24.0 & \\
\hline Tumor differentiation & & & 0.212 \\
\hline Well/median differentiated & 2 & - & \\
\hline $\begin{array}{l}\text { Poorly differentiated/mucinous } \\
\text { or signet ring cell carcinoma }\end{array}$ & 34 & 24.0 & \\
\hline \multicolumn{4}{|l|}{ Pre-neoadjuvant chemotherapy } \\
\hline TNM & & & 0.092 \\
\hline IB & 3 & - & \\
\hline II & 25 & 57.5 & \\
\hline III & 8 & 17.0 & \\
\hline ypT stage & & & 0.150 \\
\hline $\mathrm{T} 1-2$ & 9 & - & \\
\hline T3-4 & 27 & 23.0 & \\
\hline ypN stage & & & 0.002 \\
\hline $\mathrm{N}-$ & 11 & - & \\
\hline $\mathrm{N}+$ & 25 & 22.0 & \\
\hline Treatment change & & & 0.863 \\
\hline Yes & 12 & 24.0 & \\
\hline No & 24 & 31.0 & \\
\hline
\end{tabular}

OS, overall survival. chemotherapy (Figure 2). No such trends were observed in the GHR $\leq 30 \%$ vs. $>30 \%$ and GHR $\leq 40 \%$ vs. $>40 \%$ groups.

\section{Discussion}

Nearly $70 \%$ of patients with locally advanced gastric cancer respond to preoperative chemotherapy regimens, such as FU plus cisplatin (FP) (14), S-1 plus cisplatin (SP) $(15,16)$, FOLFOX $(17,18)$, or XELOX (19). In other words, approximately one-third of the patients may not respond to chemotherapy, which necessitates the use of a modified postoperative regimen. However, criteria to screen and find patients who do not benefit from preoperative chemotherapy have not been established, making it difficult to develop standardized postoperative treatment strategies. Until now, the same chemotherapy regimens were used before and after surgery in most clinical trials $(2,3,20)$. Thus, developing reliable and practical criteria is essential for the screening, identification, and development of alternative treatment strategies for patients who do not benefit from preoperative chemotherapy.

Graded (from 0\% to 100\%) histologic response provides a visual and objective method for evaluating the efficacy of neoadjuvant chemotherapy. In 2003, Becker et al. (8) proposed a classification of histopathologic regression for primary tumors, and in 2011, the Japanese Gastric Association updated its histological evaluation criteria (21). These two criteria are the most common and have been used in most studies of neoadjuvant chemotherapy for patients with gastric cancer based on histological evaluation with proven correlation with prognosis (7-10,21). However, these criteria do not define the cut-off value at which postoperative chemotherapy should be modified. While exploring the threshold of GHR, several authors have found that a GHR $\geq 50 \% /<50 \%$ could serve as a prognostic factor

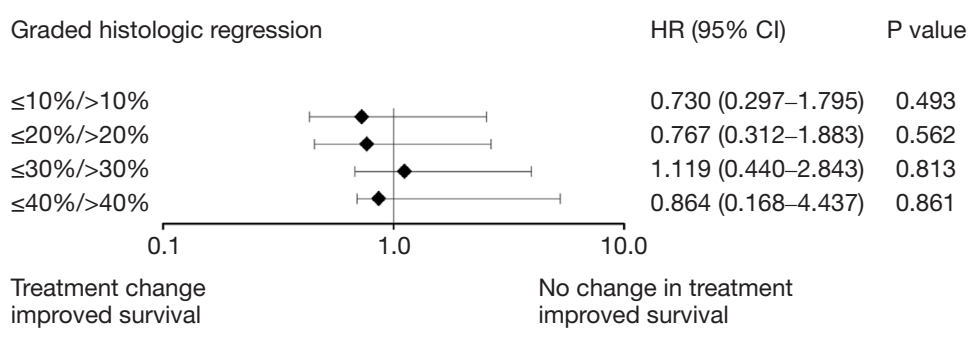

Figure 2 Hazard ratios for OS in different GHR groups. GHR, graded histologic regression; OS, overall survival. 
in resected patients after neoadjuvant chemotherapy $(6,11)$.

We referenced earlier studies $(6,11)$ and used a GHR of $<50 \%$ as the threshold for regimen modification. Although there was no significant difference in the PFS or OS between the two treatment arms, there was a clear prolongation in patients with stage III disease who received a modified regimen. The reason the subgroup analysis did not reach statistical significance is due to the small sample size. Therefore, these results suggest that the chemotherapy regimen should be promptly adjusted in patients with advanced stages and a low GHR rate. This finding was likely related, first, to the prognosis of patients with stage I or II after surgery being better than the patients with stage III. Thus, the benefits of adjuvant chemotherapy for the patients with a lower stage is not so significant compared with the patients with a higher stage who did not downstage after neoadjuvant chemotherapy. Patients with stage III, especially those with a low GHR rate, received no discernible benefit and may be insensitive to preoperative chemotherapy. Thus, these patients may benefit from modified chemotherapy. Fields et al. (22) conducted a retrospective analysis of the clinical data of 714 patients with locally advanced gastric or gastroesophageal junction adenocarcinoma who received neoadjuvant chemotherapy and found no significant difference in the relapse rate between patients with pathologic stage I or II following preoperative therapy.

Conversely, patients with pathologic stage III disease had a poor prognosis. Therefore, patients with stage III, especially those with a low GHR rate, would be better to change the ineffective neoadjuvant chemotherapy regimen. Second, most patients who received modified chemotherapy after surgery were switched to higher-intensity triplet regimens. As we know, the V325 trail (23) demonstrated that the addition of docetaxel to cisplatin and fluorouracil (DCF) not only significantly improved clinical benefit but also improved quality of life, time to progression, and overall survival compared with CF. Therefore, the patients who do not benefit from preoperative doublet chemotherapy may receive help from postoperative triplet chemotherapy. Third, all patients who received modified chemotherapy after surgery were converted to taxane-based chemotherapy, whose mechanism of action differs from that of oxaliplatin. This difference may be an essential reason for the benefit which these patients gained from modified chemotherapy.

In this study, we also used $10 \%, 20 \%, 30 \%$, and $40 \%$ GHR as the thresholds for regimen modification. The results show that the lower of the GHR rate, the more prolongation of survival would be obtained once the patients received chemotherapy modification. Therefore, patients with a low GHR rate should receive modified chemotherapy soon after surgery.

The ACTS-GC trial (24) and CLASSIC trial (25) proved the efficacy of S-1 and XELOX regimens as adjuvant chemotherapy for East Asian patients with gastric cancer who received curative surgery with D2 lymphadenectomy. Moreover, taxane has been used extensively as part of neoadjuvant chemoradiotherapy (26), chemotherapy (27-29), and adjuvant chemotherapy regimens $(30,31)$ due to its high efficacy as palliative chemotherapy for advanced gastric cancer (23). Our department (30) has previously reported a study of 32 patients with gastric cancer who received the DCF regimen as adjuvant chemotherapy. The results showed that the median disease-free survival was 17.0 months, and the 2-year disease-free survival rate was $37.5 \%$. Although the 2 -year disease-free survival rate was lower than the 3-year disease-free survival rate in the ACTS-GC trial (72.2\%) and the CLASSIC trial (74.0\%), the patients in our study generally harbored more advanced disease, as nearly $70 \%$ of the patients were diagnosed with stage III disease. Yoon et al. (31) administered the docetaxel + capecitabine + cisplatin (DXP) regimen as adjuvant chemotherapy to patients with stage IIIB-IV disease according to the AJCC 6.0; the results showed that the median relapse-free survival was 26.9 months, and the 5 -year relapse-free survival rate was $39.1 \%$. Hence, in this study, we used taxane-based chemotherapy as modified adjuvant chemotherapy after surgery. We found that this regimen was effective with controllable adverse reactions and, thus, should be used more widely in clinical practice.

This study is retrospective with small sample size. Although we were not able to confirm a survival benefit in patients with locally advanced gastric cancer who received modified chemotherapy after surgery based on a GHR $<50 \%$ after preoperative neoadjuvant chemotherapy, we found that regimen modification may be beneficial for patients with advanced disease (stage III) whose GHR was $<50 \%$ after preoperative neoadjuvant chemotherapy. These findings should be further investigated in a randomized prospective clinical trial.

\section{Acknowledgments}

We thank the patients, their families, their caregivers, and the investigators and their team members for taking part in this study. Professional statistics and data assistance were 
provided by Dr. Yanlong Li (Institute of Basic Medical Sciences, Peking Union Medical College \& Chinese Academy of Medical Sciences, Beijing, China).

Funding: This work was supported by grants from the National Natural Science Foundation of China (No. 81472785; No. 61435001) and the CAMS Innovation Fund for Medical Sciences (No. 2016-I2M-1-001).

\section{Footnote}

Conflicts of Interest: The authors have no conflicts of interest to declare.

Ethical Statement: The authors are accountable for all aspects of the work in ensuring that questions related to the accuracy or integrity of any part of the work are appropriately investigated and resolved. The study protocol was approved by the Institutional Review Board of Peking Union Medical College Hospital, Chinese Academy of Medical Sciences (No. S-613), and all the participants signed an informed consent form.

\section{References}

1. Chen $\mathrm{W}$, Zheng R, Baade PD, et al. Cancer statistics in China, 2015. CA Cancer J Clin 2016;66:115-32.

2. Cunningham D, Allum WH, Stenning SP, et al. Perioperative chemotherapy versus surgery alone for respectable gastroesophageal cancer. N Engl J Med 2006;355:11-20.

3. Ychou M, Boige V, Pignon JP. Perioperative chemotherapy compared with surgery alone for resectable gastroesophageal adenocarcinoma: an FNCLCC and FFCD multicenter phase III trial. J Clin Oncol 2011;29:1715-21.

4. Lutz MP, Zalcberg JR, Ducreux M, et al. Highlights of the EORTC St. Gallen International Expert Consensus on the primary therapy of gastric, gastroeso- phageal and oesophageal cancer-differential treatment strategies for subtypes of early gastroesophageal cancer. Eur J Cancer 2012;48:2941-53.

5. Ronellenfitsch U, Schwarzbach M, Hofheinz R, et al. Perioperative chemo(radio)therapy versus primary surgery for resectable adenocarcinoma of the stomach, gastroesophageal junction, and lower esophagus. Cochrane Database Syst Rev 2013;(5):CD008107.

6. Mansour JC, Tang L, Shah M, et al. Does graded histologic response after neoadjuvant chemotherapy predict survival for completely resected gastric cancer? Ann Surg Oncol 2007;14:3412-8.

7. Lowy AM, Mansfiled PF, Leach SD, et al. Response to neoadjuvant chemotherapy best predicts survival after curative resection of gastric cancer. Ann Surg 1999;229:303-8.

8. Becker K, Mueller JD, Schulmacher C, et al. Histomorphology and grading of regression in gastric carcinoma treated with neoadjuvant chemotherapy. Cancer 2003;98:1521-30.

9. Becker K, Langer R, Reim D, et al. Significance of histopathological tumour regression after neoadjuvant chemotherapy in gastric adenocarcinomas: a summary of 480 cases. Ann Surg 2011;253:934-9.

10. Kurokawa Y, Shibata T, Sasako M, et al., Validity of response assessment criteria in neoadjuvant chemotherapy for gastric cancer (JCOG0507-A). Gastric Cancer 2014;17:514-21.

11. Wang X, Zhao L, Liu H, et al. A phase II study of a modified FOLFOX6 regimen as neoadjuvant chemotherapy for locally advanced gastric cancer. $\mathrm{Br} \mathrm{J}$ Cancer 2016;114:1326-33.

12. Husband JE, Schwartz LH, Spencer J, et al. Evaluation of the response to treatment of solid tumours-a consensus statement of the International Cancer Imaging Society. Br J Cancer 2004; 90:2256-60.

13. NCCN Clinical Practice Guidelines in Oncology. Gastric Cancer. V1. 2016. Available online: https://www.ncen.org/ professionals/physician_gls/pdf/gastric.pdf

14. Schuhmacher C, Gretschel S, Lordick F, et al. Neoadjuvant chemotherapy compared with surgery alone for locally advanced cancer of the stomach and cardia: European Organisation for Research and Treatment of Cancer randomized trial 40954. J Clin Oncol 2010;28:5210-8.

15. Iwasaki Y, Sasako M, Yamamoto S, et al. Phase II study of preoperative chemotherapy with S-1 and cisplatin followed by gastrectomy for clinically resectable type 4 and large type 3 gastric cancers (JCOG0210). J Surg Oncol 2013;107:741-5.

16. Tsuburaya A, Mizusawa J, Tanaka Y, et al. Neoadjuvant chemotherapy with S-1 and cisplatin followed by D2 gastrectomy with para-aortic lymph node dissection for gastric cancer with extensive lymph node metastasis. $\mathrm{Br} \mathrm{J}$ Surg 2014;101:653-60.

17. Zhang J, Chen RX, Zhang J, et al. Efficacy and safety of neoadjuvant chemotherapy with modified FOLFOX7 regimen on the treatment of advanced gastric cancer. Chin Med J 2012;125:2144-50. 
18. Li ZY, Koh CE, Bu ZD, et al. Neoadjuvant chemotherapy with FOLFOX: improved outcomes in Chinese patients with locally advanced gastric cancer. J Surg Oncol 2012;105:793-9.

19. Wang Y, Yu YY, Li W, et al. A phase II trial of Xeloda and oxaliplatin (XELOX) neo-adjuvant chemotherapy followed by surgery for advanced gastric cancer patients with para-aortic lymph node metastasis. Cancer Chemother Pharmacol 2014;73:1155-61.

20. Al-Batran SE, Hofheinz RD, Pauligk C, et al. Oxaliplatin, fluorouracil, and leucovorin versus epirubicin, cisplatin, and fluorouracil or capecitabine in patients with resectable gastric or gastro-oesophageal junction adenocarcinoma (FLOT4-AIO): results from the phase 2 part of a multicentre, open-label, randomised phase 2/3 trial. Lancet Oncol 2016;17:1697-708.

21. Japanese Gastric Cancer Association. Japanese classification of gastric carcinoma: 3rd English edition. Gastric Cancer 2011;14:101-12.

22. Fields RC, Strong VE, Gönen M, et al. Recurrence and survival after pathologic complete response to preoperative therapy followed by surgery for gastric or gastrooesophageal adenocarcinoma. Br J Cancer 2011;104:1840-7.

23. Ajani JA, Moiseyenko VM, Tjulandin S,et al. Clinical benefit with docetaxel plus fluorouracil and cisplatin compared with cisplatin and fluorouracil in a phase III trial of advanced gastric or gastroesophageal cancer adenocarcinoma: the V-325 Study Group. J Clin Oncol 2007;25:3205-9.

24. Sakuramoto S, Sasako M, Yamaguchi T, et al. Adjuvant chemotherapy for gastric cancer with S-1, an oral

Cite this article as: Wang $\mathrm{X}, \mathrm{Li} \mathrm{X}$, Zhou N, Zhong D, Bai C, Zhao L. Graded histologic response after neoadjuvant chemotherapy is an optimal criterion for treatment change in patients with locally advanced gastric cancer. Ann Transl Med 2019;7(20):546. doi: 10.21037/atm.2019.09.82 fluoropyrimidine. N Engl J Med 2007;357:1810-20.

25. Bang YJ, Kim YW, Yang HK, et al. Adjuvant capecitabine and oxaliplatin for gastric cancer after D2 gastrectomy (CLASSIC): a phase 3 open-label, randomised controlled trial. Lancet 2012;379:315-21.

26. van Hagen P, Hulshof MC, van Lanschot JJ, et al. Preoperative chemoradiotherapy for esophageal or junctional cancer. N Engl J Med 2012;366:2074-84.

27. Biffi R, Fazio N, Luca F, et al. Surgical outcome after docetaxel-based neoadjuvant chemotherapy in locallyadvanced gastric cancer. World J Gastroenterol 2010;16:868-74.

28. Park I, Ryu MH, Choi YH, et al. A phase II study of neoadjuvant docetaxel, oxaliplatin, and S-1 (DOS) chemotherapy followed by surgery and adjuvant S-1 chemotherapy in potentially resectable gastric or gastroesophageal junction adenocarcinoma. Cancer Chemother Pharmacol 2013;72:815-23.

29. Yoshikawa T, Morita S, Tanabe K, et al. Survival results of a randomised two-by-two factorial phase II trial comparing neoadjuvant chemotherapy with two and four courses of S-1 plus cisplatin (SC) and paclitaxel plus cisplatin (PC) followed by D2 gastrectomy for resectable advanced gastric cancer. Eur J Cancer 2016;62:103-11.

30. Meng C, Yin H, Sun Z, et al. Adjuvant Chemotherapy with Docetaxel, Cisplatin, and Continuous-Infusion 5-Fluorouracil for Gastric Cancer: A Phase II Study. Transl Oncol 2014;7:277-83.

31. Yoon S, Yoo C, Ryu MH, et al. Phase 2 study of adjuvant chemotherapy with docetaxel, capecitabine, and cisplatin in patients with curatively resected stage IIIB-IV gastric cancer. Gastric Cancer 2017;20:182-9. 